\title{
Convexity and Hausdorff-Pompeiu Distance
}

\author{
TEMISTOCLE BÎRSAN
}

\begin{abstract}
The aim of this paper is to realize a decomposition of the usual convexity structures on metric spaces. Thus, a metric space is totally convex if and only if it satisfies the conditions (A) and (B) (Proposition 2$)$. Also, it is totally externally convex if and only if both conditions $\left(\mathrm{A}^{\prime}\right)$ and $\left(\mathrm{B}^{\prime}\right)$ are satisfied (Proposition 4$)$. Some connections between the convexity conditions $(\mathrm{A})$ and $\left(\mathrm{A}^{\prime}\right)$ and the Hausdorff-Pompeiu metric are investigated (see, for example, Corollary 3 ).
\end{abstract}

\section{Notations and Preliminaries}

Let $(X, d)$ be a metric space, and let $S(x, r)=\{y \in X ; d(x, y)<r\}$ and $S[x, r]=\{y \in X ; d(x, y) \leq r\}$ be the open and closed spheres in $X$ of center $x$ and radius $r$.

We begin with a simple remark. If $(X, d)$ is the discrete metric space (i.e. $d(x, y)=1$ if $x \neq y$, and $d(x, x)=0$ for all $x \in X)$, we have

$$
S(x, r)=\overline{S(x, r)}=\left\{\begin{array}{ll}
\{x\}, & r \leq 1 \\
X, & r>1
\end{array} \quad \text { and } \quad S[x, r]= \begin{cases}\{x\}, & r<1 \\
X, & r \geq 1 .\end{cases}\right.
$$

One can see a discontinuity in the behaviour of these spheres at $r=1$. On the other hand, the spheres $S(x, r)$ and $S[x, r]$ vary continuously in both variables $x$ and $r$, if $(X, d)$ is the Euclidean space $\mathbb{R}^{n}$ or a normed space.

Let $C L B(X)$ be the space of nonempty closed and bounded subsets of $X$. The continuity of the functions $F: X \times \mathbb{R}_{+}^{*} \rightarrow C L B(X)$ (respectively $\bar{F}: X \times \mathbb{R}_{+}^{*} \rightarrow C L B(X)$ ) defined by $F(x, r)=S[x, r]$ (respectively $\bar{F}(x, r)=$ $\overline{S(x, r)})$ is influenced by the above mentioned deficiency of the metric spaces. In general, it is necessary to assume that $(X, d)$ satisfies certain conditions of convexity to guarantee the positive results concerning the continuity of these functions [3].

Our aim is to point out the role played by convexity type conditions on a metric space in this area. For this purpose we decompose the conditions of

2000 Mathematics Subject Classification. Primary: 54 H99.

Key words and phrases. Convexity condition (A), convexity condition (B), totally convex, Hausdorff-Pompeiu metric. 
total convexity and total external convexity of a metric space into two other types of weaker convexity conditions.

Let us recall some concepts that will be needed later.

In $[4, \mathrm{p} .26]$, a metric space $(X, d)$ is said to be quasi-convex ( $Q C$-spaces) if for each $\varepsilon>0$ and each $a, b \in X, a \neq b$, there is $\delta>0$ and a point $x \in X$ such that $d(a, x)<d(a, b)-\delta$ and $d(b, x)<\varepsilon$.

E. Blanc proved [4, p.27] the equivalence of the following conditions:

1. $(X, d)$ is quasiconvex, and

2. $\overline{S(x, r)}=S[x, r]$, for all $x \in X$ and $r>0$.

Obviously, in a $Q C$-space we have $F=\bar{F}$.

The space $(X, d)$ is said to satisfy the convexity condition $(A)$, if for each $x, y \in X, x \neq y$, and each $r, s>0$ with $d(x, y)<r+s$ there exists $z \in X$ such that $d(x, z)<r$ and $d(y, z)<s[4$, p.24].

The following properties are equivalent [4, p.46]:

1. $(X, d)$ satisfies $(\mathrm{A})$;

2. $S(S(E, \alpha), \beta)=S(S[E, \alpha], \beta)=S(E, \alpha+\beta)$, for $E \subset X$ and $\alpha, \beta>$ 0

3. $S[S(E, \alpha), \beta]=S[S[E, \alpha], \beta]=S[E, \alpha+\beta]$, for $E \subset X$ and $\alpha, \beta>0$.

Finally, we mention three well-known conditions of convexity.

(M) (convexity in the sense of Menger) for $x, y \in X$, with $d(x, y) \neq 0$, there exists $z \in X, x \neq z \neq y$, such that $d(x, y)=d(x, z)+d(z, y)$;

(C) (total convexity) for $x, y \in X$, with $d(x, y) \neq 0$, and $\alpha \in(0, d(x, y))$, there exists $z \in X$ such that $d(x, z)=\alpha$ and $d(x, z)+d(z, y)=$ $d(x, y)$

$\left(\mathrm{C}^{\prime}\right)$ (total external convexity) for $x, y \in X$, with $d(x, y) \neq 0$, and $\alpha>0$, there exists $z \in X$ such that $d(y, z)=\alpha$ and $d(x, y)+d(y, z)=$ $d(x, z)$.

It is easy to check that the implications $(\mathrm{C}) \Rightarrow(\mathrm{A}) \Rightarrow(\mathrm{QC})$, and $(\mathrm{C}) \Rightarrow(\mathrm{M})$ are true.

\section{Decomposition of the Total Convexity Condition}

We begin with a characterization of the condition (A).

Proposition 1. Let $(X, d)$ be a metric space. The following are equivalent:

(i) $X$ satisfies $(A)$,

(ii) for any $x, y \in X$ and $r>0$ we have

$$
d(x, S[y, r])= \begin{cases}0, & x \in S[y, r] \\ d(x, y)-r, & x \notin S[y, r] .\end{cases}
$$

Proof. (i) $\Rightarrow$ (ii). Assume that $x \notin S[y, r]$. For $u \in S[y, r]$ we have

$$
d(x, u) \geq d(x, y)-d(y, u),
$$


hence

$$
d(x, u) \geq d(x, y)-r, \quad \text { for all } u \in S[y, r] .
$$

On the other hand, for any $\varepsilon>0$ we have $d(x, y)<d(x, y)+\varepsilon<(d(x, y)-$ $r+\varepsilon)+r$, and, by condition $(\mathrm{A})$, there exists $x_{\varepsilon} \in X$ such that $d\left(x, x_{\varepsilon}\right)<$ $d(x, y)-r+\varepsilon$ and $d\left(y, x_{\varepsilon}\right)<r$, i.e.,

$$
d\left(x, x_{\varepsilon}\right)<d(x, y)-r+\varepsilon, \quad \text { and } \quad x_{\varepsilon} \in S[y, r] .
$$

By (2) and (3) we deduce that

$$
\inf \{d(x, u) ; u \in S[y, r]\}=d(x, y)-r,
$$

therefore $d(x, S[y, r])=d(x, y)-r$, for $x \notin S[y, r]$.

(ii) $\Rightarrow(\mathrm{i})$. First of all, we observe that, if " $<$ " is replaced by " $\leq$ " in one or both inequalities $d(x, z)<r$ and $d(y, z)<s$ of $(\mathrm{A})$, then we have a condition equivalent to $(\mathrm{A})$.

Let $x, y \in X, x \neq y$, and $r, s>0$ such that $d(x, y)<r+s$. We may assume, without any loss of generality, that $r, s<d(x, y)$. Then, by (ii), $d(x, S[y, s])=d(x, y)-s$. Consequently, $d(x, S[y, s])<r$, and there exists $z \in S[y, s]$ such that $d(x, z)<r$. So, there exists $z \in X$ such that $d(x, z)<r$ and $d(y, z) \leq s$, i.e., (A) is verified, and the proof is complete.

Remark. $\quad 1)$ It is easy to see that if in the left side of (1), we place the open sphere $S(x, r)$, then the result remains valid.

2) Convexity in the sense of Menger and condition (A) are independent.

Indeed, let $X=[0,1] \times[0,1] \backslash\{(x, x) ; x \in(0,1)\}$ and $d$ the Euclidean metric on $X$. It is easy to see that $(X, d)$ verifies $(\mathrm{A})$, but $(\mathrm{M})$ is not satisfied (for the points $(0,0),(1,1) \in X$ is does not exists any point in $X$ for which the required equality is valid).

For the reverse assertion, let us consider $X=[0,1) \cup(2,3]$ equipped with Euclidean metric. One can verify that $(X, d)$ satisfies the property $(\mathrm{M})$, but not $(\mathrm{A})$. We leave the details to the reader.

Now, we need a new condition: the metric space $(X, d)$ is said to satisfy the condition $(B)$ if for any $x, y \in X$ and $r>0$ there exists $z \in S[y, r]$ such that $d(x, z)=d(x, S[y, r])$.

The following assertion gives us a decomposition of the total convexity.

Proposition 2. The metric space $(X, d)$ is totally convex if and only if both conditions $(A)$ and $(B)$ are satisfied.

Proof. Necessity is easy. For the sufficiency part, let $x, y$ be two different points in $X$ and $\lambda \in(0, d(x, y))$. Obviously, $y \notin S[x, \lambda]$. By $(\mathrm{A})$, it follows that $d(y, S[x, \lambda])=d(x, y)-\lambda$. Also, in view of $(\mathrm{B})$, there exists a point $z \in S[x, \lambda]$ such that $d(y, z)=d(y, S[x, \lambda])$. Hence, in our hypotheses, we have

$$
d(x, y) \leq d(x, z)+d(z, y) \leq \lambda+(d(x, y)-\lambda)=d(x, y)
$$


Consequently, we obtain $d(x, z)=\lambda$ and $d(x, y)=d(x, z)+d(z, y)$, and the total convexity of $(X, d)$ is established.

\section{Decomposition of the Total External Convexity Condition}

We use a procedure analogous to that in Section 2.

Let us start with two definitions.

The metric space $(X, d)$ is said to satisfy the condition $\left(\mathrm{A}^{\prime}\right)$ if for each $x, y \in X, x \neq y$, and each positive real numbers $r, s$ with $r-s<d(x, y) \leq r$ there is $z \in X$ such that $d(x, z)>r$ and $d(z, y)<s$.

Remark. 1) It can be easily verified that, if " $d(z, y)<s$ " is replaced by " $d(z, y) \leq s$ ", we have a condition equivalent to $\left(\mathrm{A}^{\prime}\right)$.

2) The condition (A) and $\left(\mathrm{A}^{\prime}\right)$ have a simple geometric interpretation. (A) guarantees the existence of a common point of the open spheres $S(x, r)$ and $S(y, s)$ (or, equivalently, $S[x, r]$ and $S[y, s]$ ), whereas $\left(\mathrm{A}^{\prime}\right)$ shows the existence of a point in $S(y, s)$ (or $S[y, s]$ ) which is external to the closed sphere $S[x, r]$.

Also, $(X, d)$ is said to satisfies the conditions $\left(\mathrm{B}^{\prime}\right)$ if for any $x, y \in X$ and $r>0$ there exists $z \in S[y, r]$ such that $d(x, z)=\sup \{d(x, u) ; u \in S[y, r]\}$.

The following two propositions are similar to the Propositions 1 and 2.

Proposition 3. Let $(X, B)$ be a metric space. The following are equivalent:

(i) the condition $\left(A^{\prime}\right)$ is satisfied;

(ii) for any $x, y \in X$ and $r>0$ we have

$$
\sup \{d(x, u) ; u \in S[y, r]\}=d(x, y)+r .
$$

Proof. (i) $\Rightarrow$ (ii). Indeed,

$$
d(x, u) \leq d(x, y)+r, \quad \text { for all } u \in S[y, r] .
$$

Let $x \neq y$ and $\varepsilon>0$ be given with $\varepsilon<r$. We can write $d(x, y)>$ $d(x, y)-\varepsilon=(d(x, y)+r-\varepsilon)-r$ and so, by hypothesis, there exists $z \in X$ such that $d(x, z)>d(x, y)+r-\varepsilon$ and $d(y, z)<r$, that is

$$
d(x, z)>(d(x, y)+r)-\varepsilon, \quad \text { and } \quad z \in S[y, r] .
$$

Finally, combining (5) and (6), we obtain the relation (4).

(ii) $\Rightarrow$ (i). Let $x, y \in X, x \neq y$, be given and let $r, s>0$ be such that $r-s<d(x, y) \leq r$. By our hypothesis,

$$
\sup \{d(x, u) ; u \in S[y, s]\}=d(x, y)+s<r,
$$

hence there exists $z \in S[y, s]$ with $d(x, z)>r$, i.e., $d(x, z)>r$ and $d(y, z) \leq$ $s$. Therefore, the condition $\left(\mathrm{A}^{\prime}\right)$ is valid. This completes the proof.

Proposition 4. A metric space $(X, d)$ is totally externally convex if and only if it satisfies the conditions $\left(A^{\prime}\right)$ and $\left(B^{\prime}\right)$. 
Proof. For the "if" part, let $x, y \in X, x \neq y$, and $\lambda>0$ be given. In view of $\left(\mathrm{A}^{\prime}\right)$, we have $\sup \{d(x, u) ; u \in S[y, \lambda]\}=d(x, y)+\lambda$. On the other hand, by $\left(\mathrm{B}^{\prime}\right)$, there exists $z \in S[y, \lambda]$ such that $d(x, z)=\sup \{d(x, u) ; u \in S[y, \lambda]\}$.

Consequently, there is $z \in S[y, \lambda]$ such that $d(x, z)=d(z, y)+\lambda$. From $d(x, y)+\lambda=d(x, z) \leq d(x, y)+d(y, z) \leq d(x, y)+\lambda$, we deduce that $d(y, z)=\lambda$, and it follows that $d(x, z)=d(x, y)+d(y, z)$. Therefore $X$ is totally externally convex.

The "only if" part is trivial.

\section{Hausdorff-Pompeiu Distance of Closed Spheres}

In what follows, $e(A, B)$ and $H(A, B)$ denote the excess of $A$ over $B$ and the Hausdorff-Pompeiu distance between $A$ and $B$, respectively. Under the conditions $(\mathrm{A})$ and $\left(\mathrm{A}^{\prime}\right)$ we will give a simple and natural expression for the Hausdorff-Pompeiu distance of two closed spheres. Now, we begin with a preliminary result.

Proposition 5. Let $(X, B)$ be a metric space. The following are true:

(i) condition (A) implies that if $S\left[x_{1}, r_{1}\right] \subset S\left[x_{2}, r_{2}\right]$, then

$$
e\left(S\left[x_{1}, r_{1}\right], S\left[x_{2}, r_{2}\right]\right)=0,
$$

and, if $S\left[x_{1}, r_{2}\right] \not \subset S\left[x_{2}, r_{2}\right]$, then

$$
e\left(S\left[x_{1}, r_{1}\right], S\left[x_{2}, r_{2}\right]\right) \leq d\left(x_{1}, x_{2}\right)+r_{1}-r_{2}
$$

(the cases $r_{1}=0$ and/or $r_{2}=0$ are not excluded);

(ii) if the relation (7) holds, then $X$ satisfies (A).

Proof. (i) Let $S_{1}$ and $S_{2}$ denote the closed spheres $S\left[x_{1}, r_{1}\right]$ and $S\left[x_{2}, r_{2}\right]$, respectively. The first part is trivial. To show the second part of (i), we use Proposition 1. Indeed, if $S_{1} \not \subset S_{2}$ we have

$$
\begin{aligned}
e\left(S_{1}, S_{2}\right) & =\sup \left\{d\left(x, S_{2}\right) ; x \in S_{1}\right\}=\sup \left\{d\left(x, S_{2}\right) ; x \in S_{1} \backslash S_{2}\right\}= \\
& =\sup \left\{d\left(x, x_{2}\right)-r_{2} ; x \in S_{1} \backslash S_{2}\right\} \leq \\
& \leq \sup \left\{d\left(x, x_{1}\right)+d\left(x_{1}, x_{2}\right)-r_{2} ; x \in S_{1} \backslash S_{2}\right\} \leq \\
& \leq d\left(x_{1}, x_{2}\right)+r_{1}-r_{2} .
\end{aligned}
$$

(ii) Assume that $x \in S[y, r]$. According to our hypothesis, we have

$$
d(x, S[y, r])=e(\{x\}, S[y, r])=e(S[x, 0], S[y, r]) \leq d(x, y)-r,
$$

hence $d(x, S[y, r]) \leq d(x, y)-r$. Combining this fact with the relation (2) in Section 2, we obtain that $d(x, S[y, r])=d(x, y)-r$. In view of Proposition 1, the space $X$ fulfills (A).

Corollary 1. If the metric space $(X, d)$ verifies the condition $(\mathrm{A})$, then

$$
H\left(S\left[x_{1}, r_{1}\right], S\left[x_{2}, r_{2}\right]\right) \leq d\left(x_{1}, x_{2}\right)+\left|r_{1}-r_{2}\right|,
$$

for all $x_{1}, x_{2} \in X$ and $r_{1}, r_{2} \in \mathbb{R}_{+}$. 
Proof. In view of (7), we can write

$$
\begin{aligned}
H\left(S\left[x_{1}, r_{1}\right], S\left[x_{2}, r_{2}\right]\right) & =\max \left\{e\left(S\left[x_{1}, r_{1}\right], S\left[x_{2}, r_{2}\right]\right), e\left(S\left[x_{2}, r_{2}\right], S\left[x_{1}, r_{1}\right]\right)\right\} \leq \\
& \leq \max \left\{d\left(x_{1}, x_{2}\right)+r_{1}-r_{2}, d\left(x_{1}, x_{2}\right)+r_{2}-r_{1}\right\}= \\
& =d\left(x_{1}, x_{2}\right)+\left|r_{1}-r_{2}\right| .
\end{aligned}
$$

Remark. The inequalities (7) and (8) can be strict. For example, in the Euclidean space $X=[0,1]$ we have

$$
H\left(S\left[0, \frac{1}{2}\right], S\left[1, \frac{1}{2}\right]\right)=e\left(S\left[0, \frac{1}{2}\right], S\left[1, \frac{1}{2}\right]\right)=\frac{1}{2},
$$

but $d\left(x_{1}, x_{2}\right)+\left|r_{1}-r_{2}\right|=1+0=1$.

Corollary 2. Let $(X, d)$ satisfy condition $(\mathrm{A})$, and $C L B(X)$ be equipped with the Hausdorff-Pompeiu uniformity $\mathcal{U}_{H}$. Then, the functions $F$ and $\bar{F}$ (see Section 1) coincide and they are $\mathcal{U}_{H}$-uniformly continuous.

Proof. According to our hypothesis, $\overline{S(x, r)}=S[x, r]$, for all $x \in X$ and $r>0$, hence $F=\bar{F}$.

Now, let $\varepsilon>0$ be given. For any $x, x^{\prime} \in X$ and $r, r^{\prime} \in \mathbb{R}_{+}^{*}$ such that $d\left(x, x^{\prime}\right)<\frac{\varepsilon}{2}$ and $\left|r-r^{\prime}\right|<\frac{\varepsilon}{2}$, we have

$$
H\left(S[x, r], S\left[x^{\prime}, r^{\prime}\right]\right) \leq d\left(x, x^{\prime}\right)+\left|r-r^{\prime}\right|<\frac{\varepsilon}{2}+\frac{\varepsilon}{2}=\varepsilon,
$$

therefore $F$ is $\mathcal{U}_{H}$-uniformly continuous.

If we wish that (7) and (8) are valid with equality signs, then an additional hypothesis is needed.

Proposition 6. In a metric space $(X, d)$ the following are equivalent:

(i) $(X, d)$ verifies $(\mathrm{A})$ and $\left(\mathrm{A}^{\prime}\right)$;

(ii) for any couple closed spheres in $X$, we have

$$
e\left(S\left[x_{1}, r_{1}\right], S\left[x_{2}, r_{2}\right]\right)= \begin{cases}0, & S\left[x_{1}, r_{1}\right] \subset S\left[x_{2}, r_{2}\right], \\ d\left(x_{1}, x_{2}\right)+r_{1}-r_{2}, & S\left[x_{1}, r_{1}\right] \not \subset S\left[x_{2}, r_{2}\right] .\end{cases}
$$

Proof. (i) $\Rightarrow$ (ii). As in the proof of the Proposition 5, we denote by $S_{1}$ and $S_{2}$ the closed spheres $S\left[x_{1}, r_{1}\right]$ and $S\left[x_{2}, r_{2}\right]$. If $S_{1} \not \subset S_{2}$, in view of (A) we obtain, in the same way, that

$$
\begin{aligned}
e\left(S_{1}, S_{2}\right) & =\sup \left\{d\left(x, x_{2}\right)-r_{2} ; x \in S_{1} \backslash S_{2}\right\}= \\
& =\sup \left\{d\left(x_{2}, x\right) ; x \in S_{1}\right\}-r_{2} .
\end{aligned}
$$

Next, in the presence of $\left(\mathrm{A}^{\prime}\right)$, and applying Proposition 3, we obtain

$$
e\left(S_{1}, S_{2}\right)=d\left(x_{1}, x_{2}\right)+r_{1}-r_{2},
$$

which is the required equality.

(ii) $\Rightarrow$ (i). Let $x \in X$ and a closed sphere $S[y, r]$ be given. First, we prove (A). We observe that $e(\{x\}, S[y, r])=d(x, S[y, r])$. On the other hand, if 
$x \notin S[y, r]$ and, by our hypothesis, we have $e(\{x\}, S[y, r])=d(x, y)-r$. Hence, $d(x, S[y, r])=d(x, y)-r$ if $x \notin S[y, r]$, and we can use Proposition 1 to conclude the proof of (A).

Let us prove $\left(\mathrm{A}^{\prime}\right)$. We have

$$
e(S[y, r],\{x\})=\sup \{d(x, z) ; z \in S[y, r]),
$$

and, by the hypothesis,

$$
e(S[y, r],\{x\})=d(x, y)+r .
$$

Thus,

$$
\sup \{d(x, z) ; z \in S[y, r]\}=d(x, y)+r,
$$

and, by Proposition 3, this is equivalent to $\left(\mathrm{A}^{\prime}\right)$.

Corollary 3. In a metric space $(X, d)$ which verifies the conditions $(A)$ and $\left(A^{\prime}\right)$, the equality

$$
H\left(S\left[x_{1}, r_{1}\right], S\left[x_{2}, r_{2}\right]\right)=d\left(x_{1}, x_{2}\right)+\left|r_{1}-r_{2}\right|
$$

holds true for all $x_{1}, x_{2} \in X$ and $r_{1}, r_{2} \in \mathbb{R}_{+}$.

Let $\mathcal{S}$ be the set of all closed spheres of the metric space $(X, d)$. Furthemore, on the set $X \times \mathbb{R}_{+}$we define the metric $\delta$ by

$$
\delta\left(\left(x_{1}, r_{1}\right),\left(x_{2}, r_{2}\right)\right)=d\left(x_{1}, x_{2}\right)+\left|r_{1}-r_{2}\right| .
$$

Corollary 4. If the metric space $(X, d)$ verifies the conditions $(A)$ and $\left(A^{\prime}\right)$, then the spaces $(\mathcal{S}, H)$ and $\left(X \times \mathbb{R}_{+}, \delta\right)$ are isometric.

Proof. The function $f: \mathcal{S} \rightarrow X \times \mathbb{R}_{+}$given by $f(S[x, r])=(x, r)$ is an isometry of these spaces.

\title{
REFERENCES
}

[1] R. Bantegnie, Sur certains espaces métriques, Indag. Math., 29 (1967), 74-75.

[2] G. Beer, Topologies on closed and closed convex sets, Kluwer Academic Publishers, 1993.

[3] T. Bîrsan, Hyperspaces of spheres and Pompeiu-Hausdorff distance, Lucrările Conferinţei S.S.M.R., ed. a III-a, Craiova (Romania), vol. I, 1999, 25-33.

[4] E. Blanc, Les espaces métriques quasi-convexes, Ann. Sci. École Norm. Sup., III Sér., 55 (1938), 1-82.

\author{
Temistocle Bîrsan \\ Department of Mathematics \\ Technical University "Gh. Asachi" \\ OF IAŞI \\ 700506 - IAŞI \\ ROMAnia \\ E-mail address: t_birsan@yahoo.com
}


